\title{
THE TOYOTA KATA METHODOLOGY FOR MANAGING THE MATURITY LEVEL OF LAST PLANNER® SYSTEM
}

\author{
Fernando Perez-Apaza ${ }^{1}$, Andre Ramírez-Valenzuela ${ }^{2}$, and Juan D. Perez-Apaza ${ }^{3}$
}

\begin{abstract}
The implementation of the Last Planner® System (LPS) generates reliable production flows in construction projects and improves the competitiveness of companies that adopt the system. Research shows a greater number of implementations in construction companies and also recognizes that the effectiveness of LPS in projects is not achieved due to partial, short-term implementations, and without continuous feedback. This paper describes a proposal for managing the LPS maturity level with the objective of implementing all the components of the methodology and developing the project organization. It proposes the use of a method based on the LPS maturity model proposed by the Lean Construction Institute and the Toyota Kata methodology, described by Myke Rother, to help organizations achieve improvement actions. The proposed methodology was evaluated in a case study and the results were compared based on literature regarding the level of adoption of the organizations implementing the LPS components. The results and indicators obtained were compared with studies on the implementation of LPS in projects.
\end{abstract}

\section{KEYWORDS}

Last Planner® System, lean construction, continuous improvement, toyota kata, maturity.

\section{INTRODUCTION}

The Last Planner® System (LPS) has been successfully implemented in construction to increase planning reliability, improve production performance, and create a predictable workflow (Hamzeh 2009). LPS is also considered as the gateway for the adoption of Lean culture in organizations. (Fauchier et. al 2013).

For Ballard and Tommelein (2016), the LPS is a system of interconnected parts and omitting one of the parts destroys the ability of the system to perform its functions. However, studies by Daniel (2017) and Lagos (2017) show that LPS implementations are short-term and partial with the components with the highest adoption being: Weekly work planning, Analysis of causes of non-compliance, and Percent Plan Complete (PPC). It also requires a human component as described by Fernandez-Solis et al. (2018) in their

Civil Engineer, National University of Engineering, Perú, jfperez@ uni.pe, orcid.org/0000-0003-03229110

2 Chief Executive Officer at Sonder Hub, Lima, Perú, raramirezv@uni.pe, orcid.org/0000-0003-4585$\underline{9946}$

3 Civil Engineer, Peruvian Union University, Perú, judipeap@ gmail.com, orcid.org/0000-0003-2277$\underline{3189}$ 
study on challenges in LPS implementation where they mention: lack of training, resistance to change, lack of leadership, and lack of experience in use.

The frameworks to obtain benefits from the implementation of LPS, (e.g. Hamzeh 2011; Ballard 2016; Daniel 2017; Mossman 2017), are divided into three stages: First stage (before the start of the project), in which the seeks to involve the senior managers of the company, configure the LPS for the project and have the necessary resources; second stage (during project execution), start with a pilot test, show the benefits of LPS, involve, train and empower team staff, it is also important to measure the effectiveness of the implementation and improve it; third stage (after project closure), manage knowledge for future projects and contribute to the community by publishing their best practices. During these stages, it is important to perform implementation maturity measurements to make decisions and improve the system (Daniel 2017). In the second stage, the teams initiate the application of LPS in the projects and require methodologies that achieve the success of the implementation.

The methodology for proper implementation should include tools to measure the level of implementation of LPS (Ballard and Tommelein 2016; Daniel 2017). In the literature review, three assessment proposals have been identified: the first, proposed by the GEPUC, which proposes the degree of implementation of LPS based on the Planning Best Practice (PBP) (Lagos 2017); the second, proposed by Daniel (2017), who proposes to use the assessment based on the PBP and assessing the implementation of LPS on projects developed by Lean Project Consulting; the third, proposed by the Lean Construction Institute (LCI 2016), which proposes an assessment of the level of maturity of LPS and other aspects for the adoption of the Lean culture in the organization. It also establishes a measure of progress based on maturity levels, which brings a greater degree of objectivity to the results (Nesensohn 2014).

The LCI has developed the "LCI Lean IPD Health and Maturity Assessment Approach" which measures team performance as well as Lean tools and practices and serves as a line of base propose improvement strategies (LCI 2016). Within this evaluation there is a section dedicated to LPS-LCI that consists of 8 components:

- Project team training: seeks consistency, discipline, coordination, efficiency and performance of the training in the team and in the project;

- Master planning: Long-term plan that seeks to comply with the contractual terms of the project, considering important milestones, in addition to allow to establish the project phases;

- Phase Planning / Pull Planning: Long-term plan of the project phases (the phase can be a period of time or a group of activities that lead to the achievement of a defined objective / milestone that releases a series of new works.), is elaborated based on the master planning in a collaborative way and following the "Pull" thought;

- Make ready planning: Intermediate plan that guarantees that the works can be done as planned, for this the lookahead and the analysis of limitations is executed based on planning by phases;

- Weekly work planning: Short-term plan that involves the last planners in the execution of what is released from the make ready planning.

- Daily commitment management (daily huddle): evaluation of daily commitments, on its compliance and the restrictions that did not allow its compliance; 
- Metrics \& Reporting: Measurements and displays of the variation of the Percent Plan Complete (PPC), Percent Constraints Removed (PCR), Tasks Anticipated (TA) and Tasks Made Ready (TMR)

- Assessment \& Continuous improvement: Team ability to make positive improvements, Proper use of PPC, Variance Pareto, Constraint Log and Root Cause Analysis, plus / delta and maturity models.

Each component of LPS is divided into 6 maturity levels (from level 0 to 5) that describe the conditions that must be met to determine the maturity of the project, the general description of each level is shown in Figure 2: .

Also, a methodology is necessary that incorporates and trains the project team to manage an effective implementation in constant evaluation and improvement (Ballard and Tommelein 2016). The following methodologies were identified: Kaizen events, Plan-Do-Check-Act (PDCA) Rapid Cycles, A3 Thinking, Toyota Kata (TK). These methodologies have a systematic approach to solving problems, unlike the others, TK proposes coaching cycles in which the last executors in the improvements are included, who within the LPS are known as the ultimate planners. Rother (2009) calls the improvement pattern carried out in the Toyota company as TK, it is a methodology that allows a cultural and sustainable change during the application of improvements in search of overcoming a challenge. The TK describes two patterns:

- Improvement Kata: Focused on taking steps to face a challenge, this pattern comprises four steps: (1) understanding the challenge, in which an objective is drawn based on the company's vision; (2) understand the current condition, define the current process metrics; (3) establish the target condition, define improvement metrics for the process; (4) experiment towards the target condition, progressively identifying the obstacles that prevent reaching the objective and executing actions until it is overcome.

- Coaching Kata: Focused on achieving Improvement Kata sustainability through Plan-Do-Check-Act (PDCA) cycles, this pattern includes developing teams through coaching sessions and those who answer these questions: What is my target condition? What is my current condition now? What obstacles prevent you from reaching the target condition? Which one are you turning to now? What's your next step? (Start of next PDCA cycle) When can we go to see the results and learning of that step?

The research seeks to relate the maturity assessment of LPS-LCI and the TK methodology, in order to manage the maturity of LPS. The following describes a proposal that integrates both methodologies. The proposal was tested in an infrastructure project with a budget of 4 million dollars, located in the Peruvian highlands whose scope included the stage of concrete works.

\section{DEVELOPMENT OF THE PROPOSAL}

The proposal is based on the synergy of the patterns of the TK methodology and the maturity assessment of LPS-LCI, to describe the proposal, two figures are presented: Figure 1 shows a scheme in which all levels of the company (organization, projects and processes) are aligned with a vision of Lean maturity; while Figure 2 describes the steps to follow to manage the maturity of LPS within a project. The proposal will be described below. 


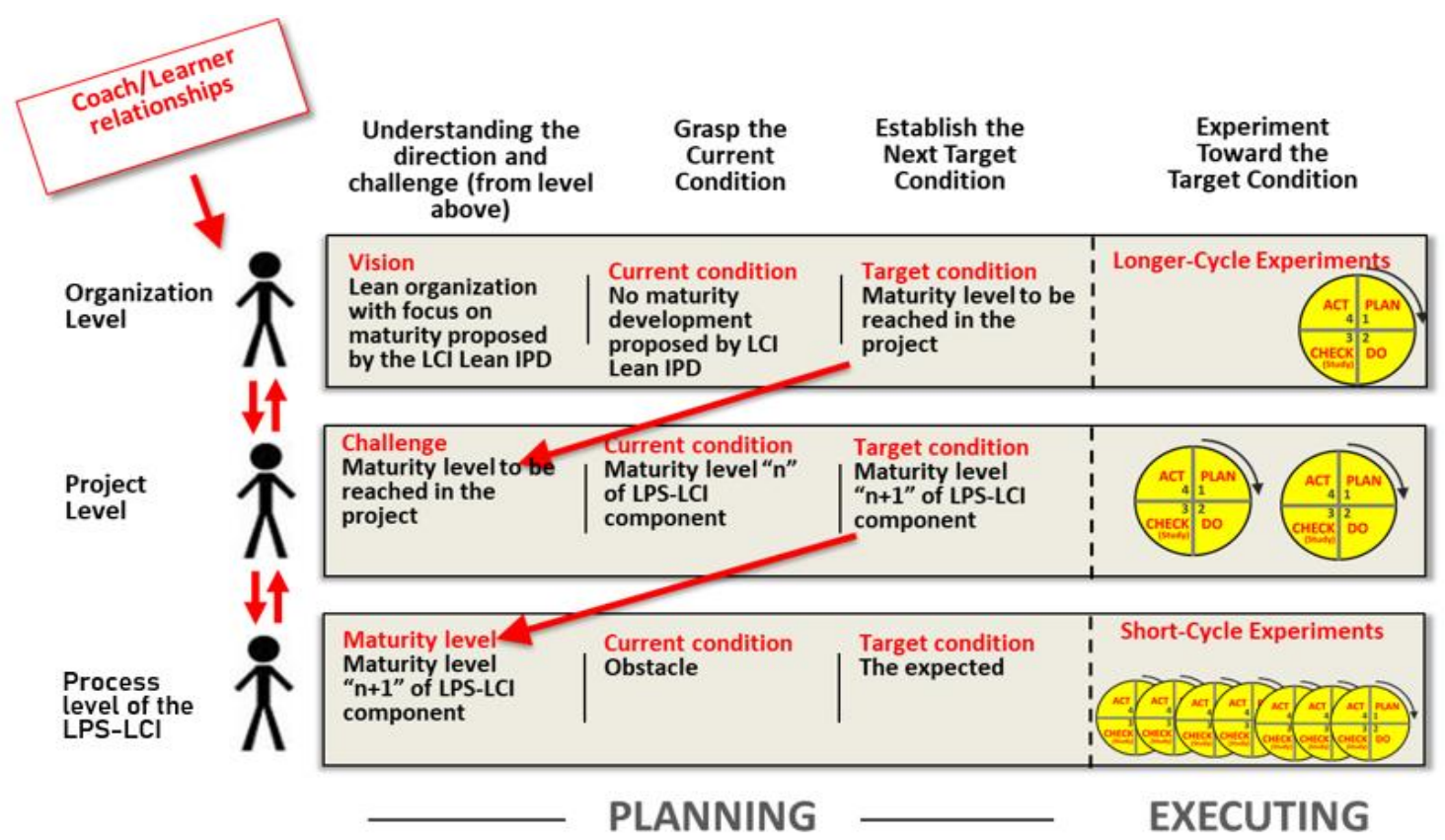

Figure 1: Manage the maturity Lean at all levels of the company

\section{Manage The Lean Maturity OF The Company}

\section{Step 1: Understand the Vision.}

At the organizational level, the vision focuses on business strategy and the adoption of the Lean culture. The three aspects of LCI are considered, which are IPD strategy (commercial), transformation change (organizational), Lean project methods \& management (operating system). The vision must be aligned by the leaders of the organization towards the leaders of the project, providing them with support as a coach and providing resources to achieve the objectives.

\section{Step 2: Grasp the Current Condition.}

Diagnostics will be made based on the LCI assessment to obtain the current condition of the maturity Lean. The radar chart (Figure 2) will be used to show the current condition. The updating of this condition is also defined based on the results obtained in the evaluation provided by the project leaders.

\section{Step 3: Establish the Next Target Condition.}

The target condition should be defined in a "Lean Maturity Assessment" meeting with the participation of organization leaders and project leaders. A target maturity level will be proposed for the projects, this will become the challenge of the leaders of each project.

\section{Step 4: Experiment Toward the Target Condition.}

During execution, obstacles are identified and actions are planned to support implementation and provide the necessary resources to the project team.

\section{Manage The Maturity Level of LPS in the Project}

\section{Step 1: Understand the Challenge.}

At the project level, the target condition proposed at the organization level becomes the challenge. The current and target condition are based on moving from level " $n$ " to " $n+1$ " according to the maturity of LPS. The evolution of the objectives is managed with Table 
1. At the process level, the project team executed the implementation by identifying the obstacles that are overcome through the use of the Storyboard and the PDCA cycles. The project leaders are the coaches of the ultimate planners.

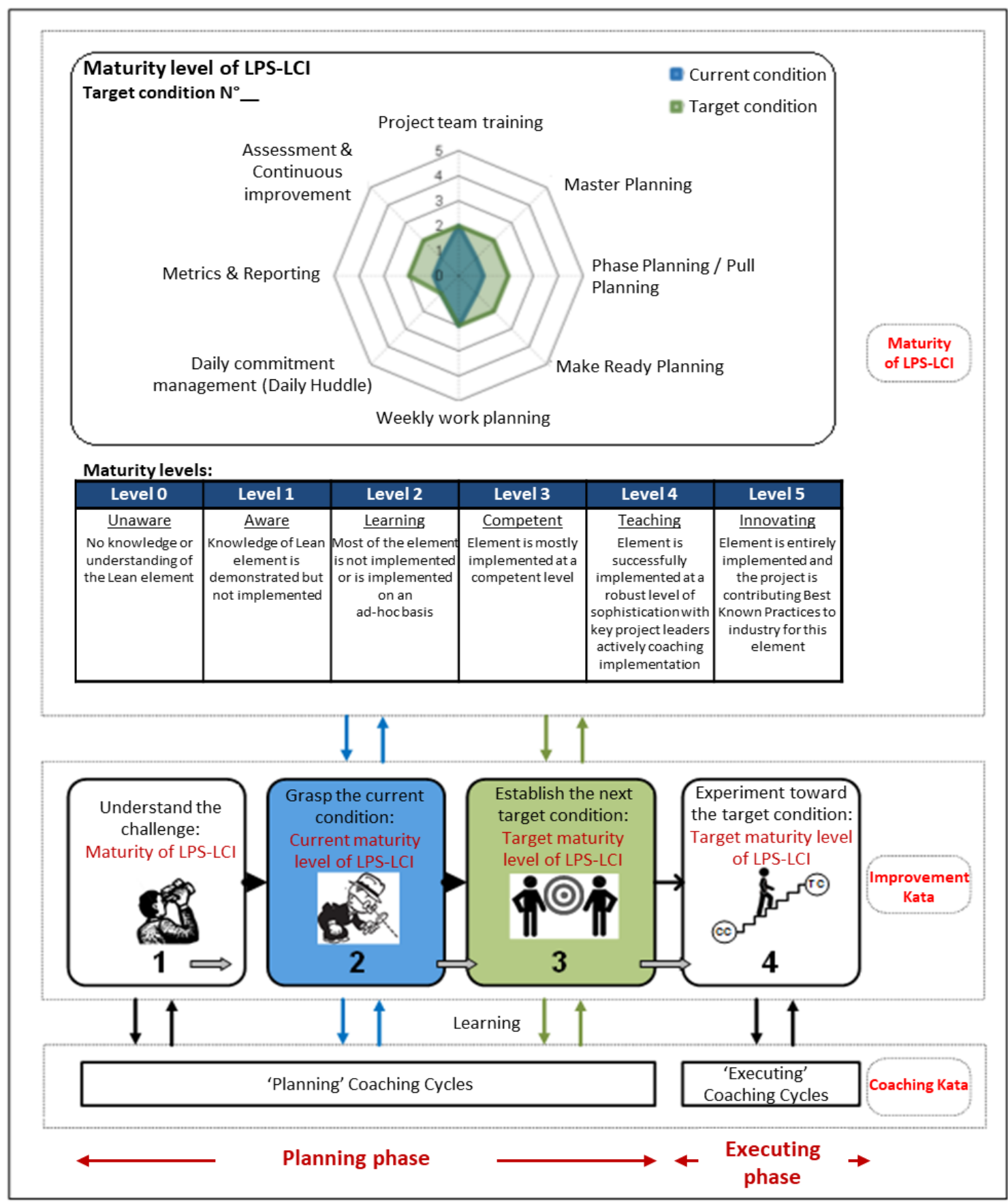

Figure 2: Manage the maturity level of LPS in the project

\section{Manage the Maturity Level of LPS in the Project}

\section{Step 1: Understand the Challenge.}

At the project level, the target condition proposed at the organization level becomes the challenge. The current and target condition are based on moving from level " $n$ " to " $n+1$ " according to the maturity of LPS. The evolution of the objectives is managed with Table 
1. At the process level, the project team executed the implementation by identifying the obstacles that are overcome through the use of the Storyboard and the PDCA cycles. The project leaders are the coaches of the ultimate planners.

\section{Step 2: Grasp the Current Condition.}

The first condition of the project is the current maturity level of LPS. While the rest of the current conditions are defined by the last reached maturity level. To assess the maturity level of LPS, evidence is needed, it can be obtained by: conversations with the project team and the last planners, corroborating the use of the tools / methods and evaluating the LPS. Subsequently, a "Maturity Assessment LPS" meeting will be convened, the objective of which will be to analyse the evidence and define the current maturity level of LPS. For a better evaluation, follow the complete guide presented by LCI (2016). The report of this assessment will be presented in a blue radar chart, as shown in Figure 2.

\section{Step 3: Establish the Next Target Condition.}

The target condition during the maturity of LPS in the project will be defined by the next level of maturity to be reached. In the aforementioned "Maturity Assessment LPS" meeting, it will be defined which components of LPS will be improve. For a better evaluation, the complete guide presented by LCI (2016). The report of this evaluation will be presented in a green radar chart, as shown in Figure 2. The target conditions that are completed will form the new current conditions.

\section{Step 4: Experiment Toward the Target Condition.}

It is based on Kata Coaching, the team is developed through PDCA cycles are executed, defining experiments whose objectives are to overcome the obstacles that arise between the current condition and the target condition of each LPS components, the planned experiments are executed and one learns from what happened. The number of obstacles to overcome, as well as the experiments to overcome them, is unknown. It should be noted that the cycles of the experiments should be short, so that, in case the response or the behaviour of the system deviates from the established direction, it is easy to take actions that can redirect the behaviour of the system. To support the interaction between the team and the coach in the PDCA learning cycles, a Storyboard should be used, documenting the maturity of LPS. The Storyboard must be printed in a minimum A3 format indicating current and target maturity levels. Figure 3 presents the format and application of the Storyboard on the site.

\section{CASE STUDY}

The case study in which the management of the maturity of LPS has been done, is a project the construction of a covered coliseum with a budget of 4 million dollars and in a construction area of $4150 \mathrm{~m}^{2}$. The construction company has experience in buildings, already having several projects executed, its philosophy is focused on the growth of its workers to achieve the delivery of quality projects, in addition the manager has knowledge about Lean Construction, but has not tried to implement. The proposal was followed by the researcher for 17 weeks, due to the stoppage of work due to weather.

\section{Manage The Lean Maturity In THE CoMPANY}

To understand the vision of Lean maturity, visits and interviews were held with the company manager, in which information was shared about the benefits and success stories 
of the implementation of LPS, it was also proposed to apply the management proposal of LPS-LCI maturity in a company project. With their approval, the organization leader defined a vision focused on the adoption of the Lean culture based in the "LCI Lean IPD health and maturity assessment" considering: IPD, Methods and Management, Transformational Change, and LPS.

To grasp the current condition, a meeting was held with the leader of the organization and project leader, the Lean maturity assessment were made to the projects, showing low maturity in all aspects.

For the target condition, it was recommended to start with the implementation of LPS since it is a methodology that is the gateway to the Lean culture, the company took on this challenge and proposed to the project that all components have at least a level 3 or competent (in Table 1).

For the experimentation towards the target condition, the organization done followups through the radar chart on a monthly basis. This information is generated by the project team and presented by the project leader. The feedback received by the leader of the organization allowed him to update the target conditions of the organization and the requirement of resources for the development of the implementation.

\section{Manage the Maturity Level of LPS in the Project}

To understand the challenge of maturity of LPS, the organization and the project leader formally communicated the decision to implement the LPS during project implementation. The project team was trained, showing the benefits and successes of LPS, an introduction to the concepts of TK and LPS, and a presentation of the proposed methodology.

To grasp the current condition, evidence was collected on the maturity status of LPS and at the "Maturity Assessment" meeting a common understanding was reached on the maturity level of LPS-LCI components. After that, the agreement is documented in a table and represented in a radar chart.

For the target condition, and being a project in which the intervention was done in full execution, it was decided to follow the implementation framework initially focused on a short-term planning, and then apply an intermediate planning and a long-term planning as recommended by Mossman (2017). This guides the team in the decision to select which component of LPS to improve and lead to a higher maturity level. Four "Maturity Assessment" meetings were hold, these are represented in

Table 1, with the components of LPS in the rows and the target conditions that were done in the project in the columns.

For the experimentation towards the target condition, from the challenge presented and established the current and target conditions, the Improvement Kata and Coaching Kata cycles began to work effectively. Following the methodology, the cycle records are presented according to the target conditions that were defined in the meetings to evaluate the maturity level of LPS. Figure 3 shows the process done to overcome an obstacle identified within the maturity of the Weekly Work Planning, the entire process is documented in a Storyboard located in the Big Room. The table called "Improvement Kata" focuses on the team to meet the target and allows identifying obstacles, while the table "Coaching Kata" allows learning from planned actions based on a dialogue between the project leader and the latest planners. 


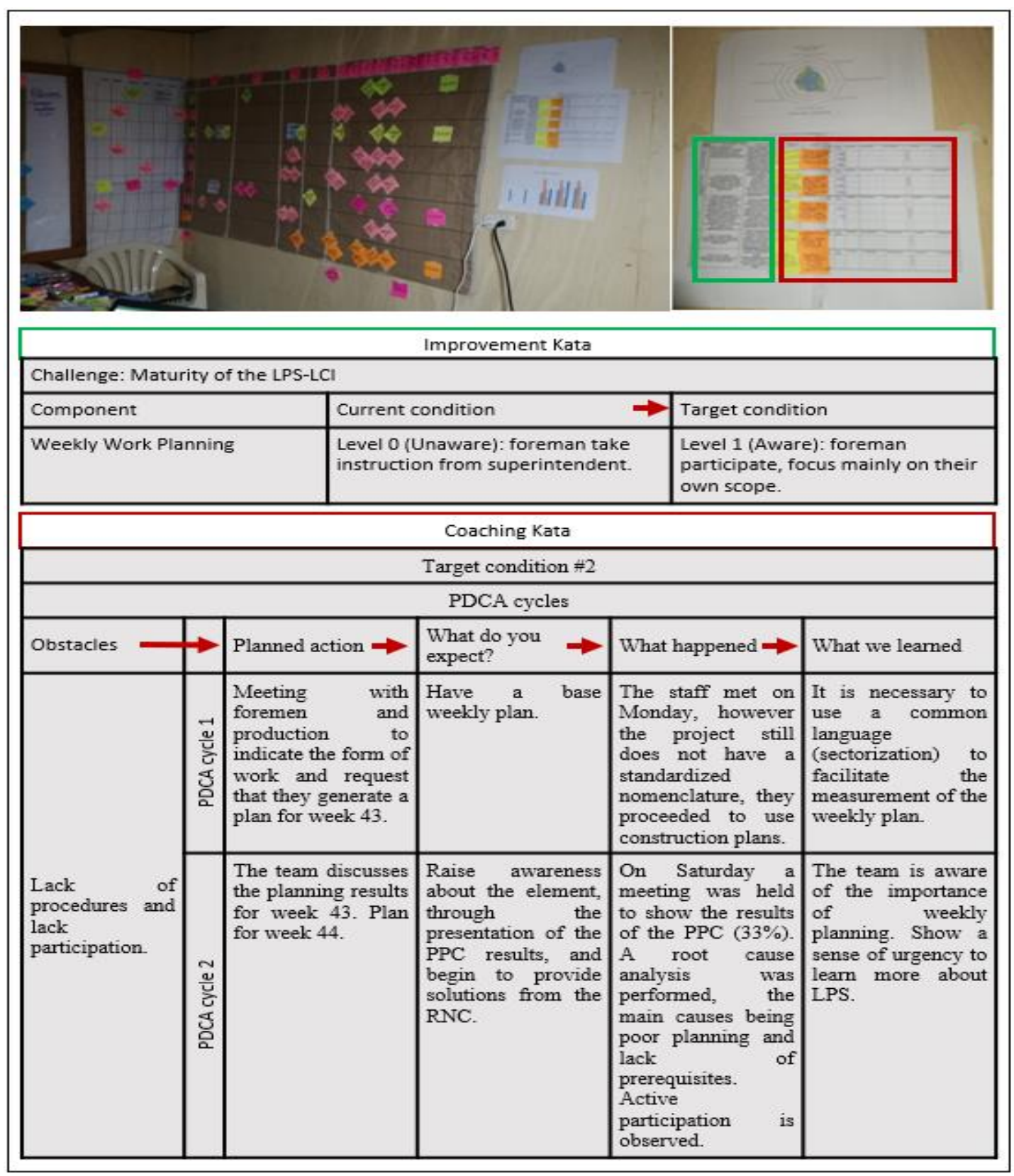

Figure 3: Example of Storyboard in the Big Room

\section{RESULTS}

Table 1 shows the development of the maturity of LPS components for the three target conditions overcome in the project. It is observed that the challenge posed at the organizational level was not met due to external factors forced the work stoppage. To reach the final maturity shown by the third target condition, it was necessary to detect 44 obstacles and execute 52 improvement actions.

Below is a comparison of the results achieved for each component with respect to the percentage of application of similar components of LPS in the studies by Daniel (2017) and Lake (2017).

- Project team training: evidence that $42 \%$ of the cases studied implemented early training; in this research it was implemented to level 3. 
- Master planning: evidence that $82 \%$ of the cases studied implemented master planning; in this research it was implemented to level 2.

- Phase planning/Pull planning: evidence that $72 \%$ of the cases studied implemented planning pull; in this research it was implemented to level 3.

- Make ready planning: evidence that $61 \%$ of the cases studied implemented lookahead, 33\% implemented constraint analysis and 19\% implemented executable work inventory; in this research it was implemented to level 3.

- Weekly work planning: evidence that $85 \%$ of the cases studied implemented weekly work planning; in this research it was implemented to level 3.

- Daily commitment management (daily huddle): evidence that $21 \%$ of the cases studied implemented daily huddle; in this research it was implemented to level 2.

- Metrics \& Reporting: evidence that $68 \%$ of the cases studied implemented PPC; in this research it was implemented to level 3.

- Assessment \& Continuous improvement: evidence that $39 \%$ of the cases studied implemented improvement actions; in this research it was implemented to level 2.

Table 1 Maturity level of LPS components defined in each target condition

\begin{tabular}{ccccc}
\hline CPS components & $\begin{array}{c}\text { Current } \\
\text { condition 0 }\end{array}$ & $\begin{array}{c}\text { Target } \\
\text { condition 1 }\end{array}$ & $\begin{array}{c}\text { Target } \\
\text { condition 2 }\end{array}$ & $\begin{array}{c}\text { Target } \\
\text { condition 3 }\end{array}$ \\
\hline Project Team Training & $\begin{array}{c}\text { Level 0: } \\
\text { Unaware }\end{array}$ & $\begin{array}{c}\text { Level 2: } \\
\text { Learning }\end{array}$ & $\begin{array}{c}\text { Level 3: } \\
\text { Competent }\end{array}$ & \\
Master Planning & Level 1: & & Level 2: & \\
Aware & & Learning & \\
Phase Planning / Pull & Level 0: & & Level 2: & Level 3: \\
Planning & Unaware & & Learning & Competent \\
Make Ready Planning & Nivel 1: & & Level 2: & Level 3: \\
& Aware & & Learning & Competent \\
Weekly Work Planning & Level 0: & Level 1: & Level 2: & Level 3: \\
Unaily Commitment & Level 0: & & Learning & Competent \\
Management (Daily Huddle) & Unaware & & Aware & Level 2: \\
& Learning \\
Metrics \& Reporting & Unaware & Level 1: & Level 2: & Level 3: \\
& Aware & Learning & Competent \\
Assessment \& Continuous & Level 0: & Level 1: & & Level 2: \\
Improvement & Unaware & Aware & & Learning \\
\hline
\end{tabular}

\section{CONCLUSIONS}

The methodology uses Maturity Assessment of LPS-LCI to diagnose the level of implementation and determining a current condition, also it allows to know the characteristics of higher levels and to establish a target condition, on the other hand the TK methodology achieves the stated condition, being the IK a guide with ordered steps that manages the improvement actions, while the CK allows a transfer of knowledge in the team members from the actions proposed to overcome an obstacle, this breaks cultural barriers and achieve a configuration of the LPS adapted to the project. The proposed 
methodology was able to implement all components of the LPS in a project, to achieve the team developed storyboards overcoming 44 obstacles and executed 52 improvement actions.

The use of the LCI Lean IPD Health and Maturity Assessment Tool enabled the organization and the project team to gain insight beyond LPS and assist in the adoption of a lean culture in the organization. Also involve the organizational team in the results of the project, which allows managing the resources necessary for the project. The proposed methodology reached level 3 in five components of LPS and level 2 in two components of LPS. Compared to other studies in which the LPS is partially implemented, with the implementation of the proposal the company achieves an implementation of all the components of the LPS and thus takes advantage of the full ability of the LPS.

For future research, it is recommended to collect data from the use of this proposal to improve measurements and make better decisions. Also update assessment based on new studies on LPS. In addition, it is proposed to use this methodology for the implementation of other fundamental aspects of the lean culture in the organization.

\section{REFERENCES}

Ballard, G. and Tommelein, I. (2016). "Current Process Benchmark for the Last Planner ${ }^{\circledR}$ System" Lean Construction Journal 2016, 57-89.

Daniel, E. I. (2017). Exploratory study into the use of Last Planner ${ }^{\circledR}$ System and collaborative planning for construction process improvement, $\mathrm{PhD}$ Dissertation, Nottingham Trent University.

Fauchier, D. and Alves, T.C.L. (2013). "Last Planner ${ }^{\circledR}$ System Is the Gateway to Lean Behaviors" Proc. Of the 21th Ann. Conf. of the Int'l Group for Lean Construction. Fortaleza, Brazil.

Fernandez-Solis, J., Porwal, V. y Lavy S. (2018). Guidelines to Address Challenges Faced by Last Planner Users and Implementers at Organizational and Project Levels. Construction Science. Accessed March 10, 2021. http://hdl.handle.net/1969.1/166276.

Hamzeh, F. (2009). Improving construction workflow- The role of production planning and control, $\mathrm{PhD}$ Dissertation, University of California at Berkeley.

Hamzeh, F. R. (2011). "The lean journey: implementing the last planner system in construction" Proc. Of the 19th Ann. Conf. of the Int'l Group for Lean Construction, Lima, Peru.

Lagos, C. (2017). Desarrollo e implementación de herramientas para el mejoramiento de la gestión de la información de last planner, Master's Thesis, Pontificia Universidad Católica de Chile.

Lean Construction Institute (2016). LCI Lean IPD Health and Maturity Assessment Tool 1.0. Accessed March 2021. https://www.leanconstruction.org/pages/learning/tools-and-technologies/.

Mossman, A. (2017). Collaborative Planning: 5+ 1crucial and Collaborative Conversations for Predictable Design \& Construction Delivery. The Change Business Ltd. https://bit.ly/3wNTtMx (15May21).

Nesensohn, C. (2014). An innovative framework for assessing lean construction maturity, PhD Dissertation, Liverpool John Moores University.

Rother, M. (2009). Toyota Kata: Managing People for Improvement, Adaptiveness and Superior Results. McGraw-Hill., United State.

Rother, M. (2016). Kata Slides \& Graphics v4.1. Accessed May 30, 2021. https://www.slideshare.net/mike734/kata-slides-graphics. 